\title{
DERIVING TOPOLOGICAL RELATIONSHIPS BETWEEN SIMPLE REGIONS WITH HOLES
}

\author{
Mark McKenney, Reasey Praing, and Markus Schneider ${ }^{\star}$ \\ Department of Computer and Information Science \& Engineering, University of Florida \\ Gainesville, Florida, USA \\ \{mm7, rpraing, mschneid\}@cise.ufl.edu
}

\begin{abstract}
Topological relationships between objects in space are of great importance in many disciplines. Due to the lack of local topological information between components, i.e. faces, in the model of topological relationships between complex regions, recently, localized topological relationships have been defined for complex regions based on the relationships between simple regions with holes. However, unlike for simple regions, topological relationships between simple region with holes are not widely implemented. Therefore, in this paper, we propose an approach to derive topological relationships between simple regions with holes based on well known topological relationships between the simple regions as their components. This will allow localized topological predicates between complex regions to be implemented using only topological relationships between simple regions. Furthermore, localized topological predicates between complex regions can be used to implement topological relationships between complex regions. Therefore, this work allows topological relationships between complex regions to be implemented using only topological relationships between simple regions.
\end{abstract}

\section{INTRODUCTION}

The exploration of relationships between spatial objects is an important topic in fields such as artificial intelligence, robotics, VLSI design, linguistics, CAD, and GIS. Object relationships can be used not only to gain information about the objects involved but also for inferring new, non-explicit information as well as creating fast access and indexing structures in spatial databases. Specifically, topological relationships have been the focus of extensive research for a long time. This research includes the design of models of topological relationships between all types of spatial objects as

\footnotetext{
* This work was partially supported by the National Science Foundation (NSF) under grant number NSF-CAREER-IIS-0347574.
} 
well as related topics like the exploration of topological relationships as a reasoning tool.

Models for topological relationships have predominantly considered simple spatial data types. A simple point object is defined as a single pair of coordinates, a simple line object is given as a non self-intersecting connected curve, and a simple region object is represented as an areal object topologically equivalent to a closed disc. A well-known model that defines the topological relationships between simple spatial objects is the 9-intersection model (9IM). The commonly known set of eight topological relationships originally defined by the 9IM between simple regions includes the relationships overlap, meet, inside, contains, coveredBy, covers, equal, and disjoint. This model was then extended to support topological relationships between complex spatial types. Roughly, a complex point is defined by a set of disjoint simple points. A complex line is composed of a set of blocks of connected simple lines. A complex region is defined as a set of one or more faces, each possibly containing holes.

The application of the 9IM to complex spatial data types has raised awareness of the global nature of the 9IM. That is, the 9IM considers the interior, exterior, and boundary point sets of the whole objects, and ignores the fact that complex spatial objects are composed of individual and separate components. As a result, local topological information regarding the relationship between individual components from each object is lost. Recently, this problem has been addressed through the introduction of localized topological relationship models, which are able to represent the topological relationships between components of complex regions, and hybrid topological relationship models, which can represent both the global and local topological relationships between complex regions (McKenney et al., 2007). However, these models are based upon topological relationships between simple regions with holes, a data type which is not typically implemented in spatial database systems. Furthermore, the complete set of topological relationships between complex regions is currently not implemented in any commercial spatial database system. Thus, the integration of local and hybrid topological relationship models, as well as the global topological relationships cannot be fully utilized in spatial systems at this time.

Although the complete set of topological relationships between complex regions, local topological predicates, and hybrid topological predicates are not fully implemented in commercial systems, implementations of the well known eight topological relationships between simple regions are commonly available. Therefore, the overall goal of this paper is to develop a method by which the topological predicates between complex regions can be characterized using only the eight topological predicates between simple regions. It has been shown that topological relationships between complex regions can be defined based on topological relationships between simple regions with holes using the localized topological relationship model. In this paper, we achieve this goal by developing a method by which topological relationships between simple regions with holes can be defined based on topological predicates between simple regions. Such a method will allow localized topological relation- 
ships between complex regions to be directly implemented on top of any system that provides the topological predicates between simple regions.

The remainder of this paper is structured as follows: Section 2 introduces related work. Section 3 demonstrates our concept of identifying topological relationships between simple regions with holes using topological relationships between simple regions. Finally, Section 4 gives conclusions and discusses future work.

\section{RELATED WORK}

In this section, we consider previous works on spatial data models including the definition of different types of regions as well as the 9-intersection model which characterizes the topological relationships between them.

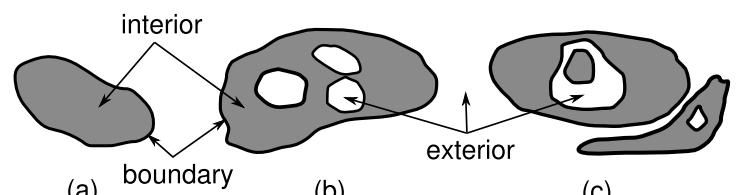

(a)

(b)

(c)

Fig. 1. A simple region (a), a simple region with holes (b), and a complex region (c).

As defined in Egenhofer and Franzosa (1991); Schneider and Behr (2006), a region consists of the interior, the boundary and the exterior point sets. Based on this definition, Figure 1 illustrates the differences between these point sets. A simple region (e.g. Egenhofer et al., 1989) is an areal object topologically equivalent to a closed disk (Figure 1a). A simple region with holes (e.g. Egenhofer et al., 1994) is made up of an outer polygon denoting its outer boundary and zero or more hole polygons representing its holes (Figure 1b). All holes must be completely contained within the outer polygon and can share a finite number of boundary points with the outer cycle and with other holes. A complex region (e.g. Schneider and Behr, 2006) is composed of faces where each face is a simple region with holes (Figure 1c).

Topological relationships between spatial objects can be defined by the 9-intersection model (e.g. Egenhofer and Franzosa, 1991; Egenhofer and Herring, 1990) by evaluating the non-emptiness of the intersection between all combinations of the interior $\left(^{\circ}\right)$, boundary $(\partial)$ and exterior $\left(^{-}\right)$of the objects involved. A $3 \times 3$ matrix with Boolean value elements, as illustrated in Figure 2, describes the topological relationship between each pair of spatial objects. Table 1 shows the 8 topological relationships between simple regions.

Originally defined for simple regions, the 9IM has been extended to handle simple regions with holes (e.g. Egenhofer et al., 1994), and complex spatial objects 


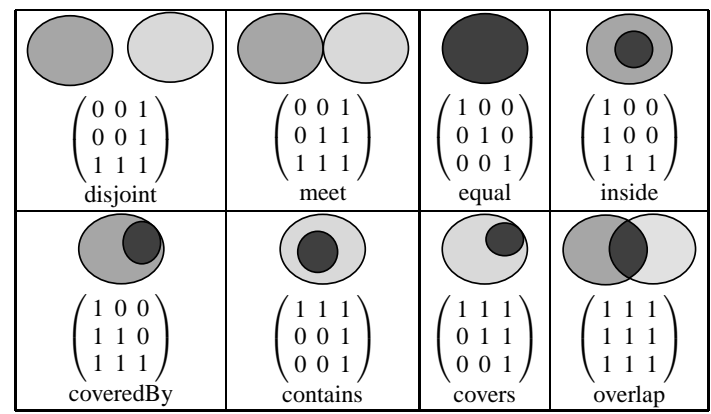

Table 1. The 8 topological relationships between simple regions.

(e.g. Clementini and Di Felice, 1996; Schneider and Behr, 2006). The model in Egenhofer et al. (1994) characterizes the topological relationships between two simple regions with holes as the conjunction of topological relationships between their underlying simple regions (each of the outer cycles and the holes is considered a simple region). For two simple regions with holes $A$ and $B$ with $n$ and $m$ holes respectively, a matrix of $(n+1)(m+1)$ elements represents the topological relationship between $A$ and $B$. This means that under this model, the number of topological relationships between two simple regions with holes is dependent on the number of holes in each region, resulting in an arbitrary number of relationships. A similar approach between composite regions can be found in Clementini et al. (1995). To avoid having an infinite set of valid topological relationships, the finite set of topological relationships between simple regions with holes based on the 9IM that is independent of the number of holes in each object has been identified in

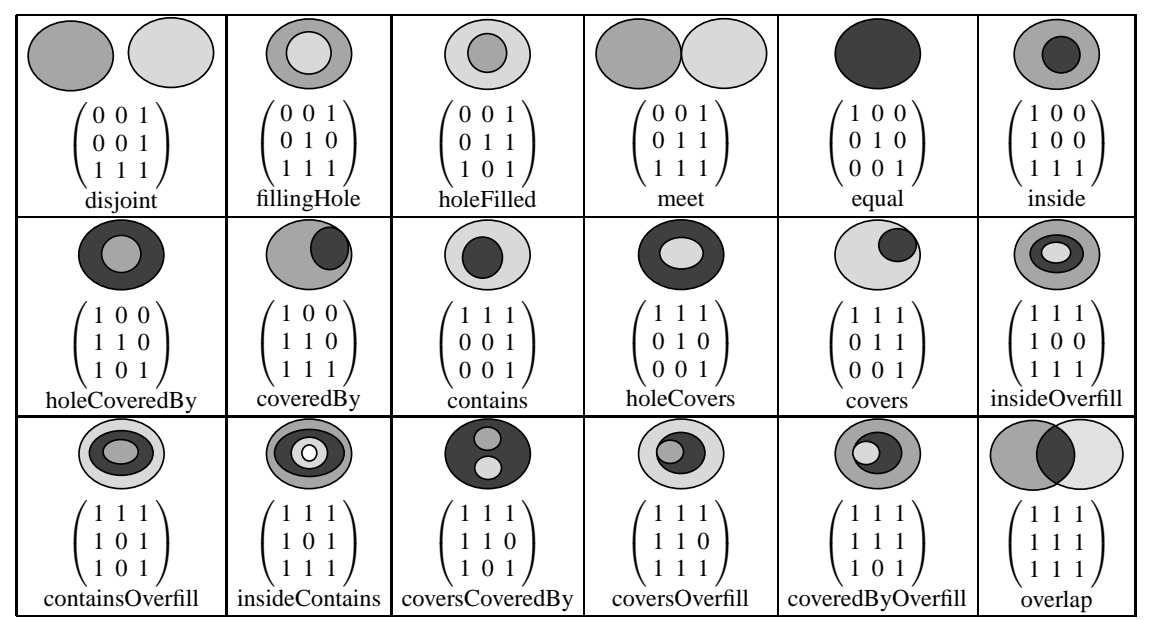

Table 2. The 18 topological predicates between simple regions with holes. 
McKenney et al. (2007). This set consists of 18 topological relationships as shown in Table 2. These relationships are used to define a model that preserves local topological relationships between complex regions while still maintaining global information. However, the approach assumes that an implementation of topological relationships between simple regions with holes exists. It turns out that this is not the case in many of today's spatial database management systems. Therefore, in this paper, we introduce a method for deriving the relationships between simple regions with holes from using only the relationships between their underlying simple regions.

$$
\left(\begin{array}{ccc}
A^{\circ} \cap B^{\circ} \neq \varnothing & A^{\circ} \cap \partial B \neq \varnothing & A^{\circ} \cap B^{-} \neq \varnothing \\
\partial A \cap B^{\circ} \neq \varnothing & \partial A \cap \partial B \neq \varnothing & \partial A \cap B^{-} \neq \varnothing \\
A^{-} \cap B^{\circ} \neq \varnothing & A^{-} \cap \partial B \neq \varnothing & A^{-} \cap B^{-} \neq \varnothing
\end{array}\right)
$$

Fig. 2. The 9-intersection matrix for topological relationships.

\section{CONSTRUCTING TOPOLOGICAL RELATIONSHIPS BETWEEN SIMPLE REGIONS WITH HOLES}

As stated in Section 2, a simple region with holes is constructed of an outer cycle and finitely many hole cycles. Each of these cycles defines the boundary of a simple region. Our approach to deriving topological relationships between simple regions with holes $A$ and $B$ is to discover the individual entries of the 9IM depicting the topological relationship between $A$ and $B$ by examining the interactions of the individual cycles of $A$ with the cycles of $B$. We then show how such interactions can be discovered using topological predicates between simple regions. This allows us to characterize a topological relationship between simple regions with holes (Table 2) using only topological predicates between simple regions (Table 1).

As a matter of notation, we indicate the 9IM representing the topological relationship between two simple regions with holes $A$ and $B$ as $M_{(A, B)}$. Furthermore, we indicate the matrix entry corresponding to whether or not the interiors of the objects intersect as $M_{(A, B)}{ }^{\circ}$, the entry corresponding to whether or not the interior of $A$ and the boundary of $B$ intersect as $M_{(A, B)}{ }^{\circ} \partial$, etc. We use the notations $\omega(A)$ and $\mathrm{\imath}(A)$ to indicate the set of simple regions formed by the outer cycle and hole cycles of a simple region with holes $A$, respectively. Note that $\omega(A)$ is a singleton set since a simple region with holes has a single outer cycle. The closure of a simple region with holes $A$ is denoted as $\bar{A}$ and is defined as the union of the boundary and interior of $A$.

Given two simple regions with holes $A$, and $B$, we must now show how to determine the values for the entries in $M_{(A, B)}$. Here, we only need to characterize the value 
for entries $M_{(A, B)}^{\circ \circ}, M_{(A, B)}{ }^{\circ} \partial, M_{(A, B)}{ }^{\circ-}, M_{(A, B)} \partial \partial$, and $M_{(A, B)} \partial^{-}$. This is because $M_{(A, B)}{ }^{--}$is always true and the characterization of entries $M_{(A, B)} \partial^{\circ}, M_{(A, B)}{ }^{-\circ}$, and $M_{(A, B)}{ }^{-} \partial$ is the same as that of entries $M_{(A, B)}{ }^{\circ} \partial, M_{(A, B)}{ }^{\circ-}$, and $M_{(A, B)} \partial^{-}$respectively with $A$ and $B$ swapped. In order to determine the entries of $M_{(A, B)}$, we make several observations about the interactions of the components of two simple regions with holes $A$ and $B$ :

Observation 1 The interiors of $A$ and $B$ intersect if the interiors of their outer cycles intersect, unless one of the outer cycles is completely contained in a hole of the opposing region. As an example, consider Figure 3. This illustrates the case when the interiors of two simple regions with holes do not intersect, even though the interiors of the simple regions defined by their outer cycles do intersect.

Observation 2 The interior of $A$ intersects the boundary of $B$ if the simple region representing any hole or outer cycle from $B$ intersects the interior of the outer cycle of $A$ and that region is not completely contained in a hole of $A$.

Observation 3 The interior of $A$ intersects the exterior of $B$ if the interior of the outer cycle of $A$ intersects the exterior of the outer cycle of $B$ or the interior of the simple region representing any hole cycle of $B$ if this hole of $B$ is not completely contained in a hole of $A$ (Note that the interior of the simple region representing a hole cycle in a simple region with holes represents the exterior of the simple region with holes).

Observation 4 The boundaries of two simple regions with holes intersect if the boundaries of any of their cycles intersect.

Observation 5 The boundary of $A$ intersects the exterior of $B$ if the boundary of the outer cycle of $A$ intersects the exterior of the outer cycle of $B$, or if the boundary of any cycle from $A$ intersects the interior of a simple region representing a hole cycle of $B$.

We state Observations 1 - 5 formally in Theorem 1.

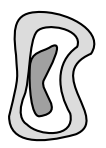

Fig. 3. Two simple regions with holes. 
Theorem 1. Let $A$ and $B$ be two simple regions with holes. The values of entries in the $9 I M$ describing their topological relationship can be written as:

(i) $\quad M_{(A, B)}{ }^{\circ \circ}=\left(\exists c \in \omega(A), \exists d \in \omega(B) \mid c^{\circ} \cap d^{\circ} \neq \varnothing\right)$

$$
\begin{aligned}
& \wedge \neg(\exists c \in \omega(A), \exists h \in \mathfrak{i}(B) \mid \bar{c} \subseteq \bar{h}) \\
& \wedge \neg(\exists j \in \mathfrak{i}(A), \exists d \in \omega(B) \mid \bar{d} \subseteq \bar{j})
\end{aligned}
$$

(ii) $M_{(A, B)}{ }^{\circ} \partial=\left(\exists c \in \omega(A), \exists d \in \omega(B) \cup \mathfrak{\imath}(B) \mid c^{\circ} \cap \partial d \neq \varnothing\right)$

$$
\wedge \neg\left(\exists h \in \mathfrak{l}(A), \exists d \in \omega(B) \mid \bar{d} \subseteq h^{\circ}\right)
$$

(iii) $M_{(A, B)}{ }^{\circ-}=\left(\exists c \in \omega(A), \exists d \in \omega(B) \mid c^{\circ} \cap d^{-} \neq \varnothing\right)$

$$
\vee\left(\exists c \in \omega(A), \exists j \in \mathfrak{i}(B), \forall h \in \mathfrak{i}(A) \mid c^{\circ} \cap j^{\circ} \neq \varnothing \wedge \bar{j} \not \bar{h}\right)
$$

(iv) $M_{(A, B)} \partial \partial=\exists c \in \omega(A) \cup \mathfrak{\imath}(A), \exists d \in \omega(B) \cup \mathfrak{\imath}(B) \mid \partial c \cap \partial d \neq \varnothing$

(v) $M_{(A, B)} \partial^{-}=\left(\exists c \in \omega(A) \cup \mathfrak{l}(A), \exists d \in \omega(B) \mid \partial c \cap d^{-} \neq \varnothing\right)$

$$
\vee\left(\exists c \in \omega(A) \cup \mathfrak{l}(A), \exists h \in \mathfrak{l}(B) \mid \partial c \cap h^{\circ} \neq \varnothing\right)
$$

Proof.

(i) The interiors of two simple regions with holes can only intersect if the simple regions represented by their outer cycles intersect. However, because holes are allowed in simple regions with holes and the interior of a simple region representing a hole is part of the exterior of the simple region with holes, then the interiors of two simple regions with holes cannot intersect if one outer cycle is completely contained in a hole of the opposing region. This follows from the definition of simple regions with holes stating that a simple region with holes contains only a single outer cycle. Therefore, the interior of a hole is entirely part of the exterior of the simple region with holes.

(ii) Note that the boundary of a simple region with holes consists of the boundaries of both the outer cycle and the hole cycles. This is in contrast to the interior of a simple region with holes which consists of the difference of the interior of the simple region representing the outer cycle, and the interiors of all simple regions representing hole cycles. Therefore, if the boundary of a simple region representing either an outer or hole cycle from $B$ intersects the interior of the outer cycle of $A$, then the boundary of $B$ intersects the interior of $A$ unless one special case occurs. This special case is when $B$ is a subset of the interior of a hole in $A$. In other words, if the outer cycle of $B$ is completely contained in the interior of a simple region representing a hole in $A$, then the boundary of $B$ cannot intersect $A$. Note that it is impossible for the intersection of the boundary of $B$ and the interior of $A$ to be completely contained in more than one hole of $A$ since this would either require two holes to meet along a boundary, or for the holes to connect as to form a second face of $A$, both of which are prohibited by the definition of simple regions with holes.

(iii) If the interior of the simple region defined by the outer cycle of $A$ intersects the exterior of the simple region defined by the outer cycle of $B$, then the boundary of $A$ intersects the exterior of $B$. This follows from the definition of simple regions. Furthermore, the interior of $A$ intersects the exterior of $B$. This follows from the fact that if the boundary of a simple region with holes intersects the exterior of a second simple region with holes, the interior of the first simple region with holes must also intersect the exterior of the second region with holes (McKenney et al., 2007). Alternatively, the interior of the simple region defined by the outer cycle of $A$ 
can intersect the interior of a hole of $B$. However, if that hole is completely contained in a hole of $A$, then the interior of $A$ will not intersect the exterior of $B$.

(iv) The boundary of a simple region with holes consists of the boundaries of all the outer cycles and the hole cycles of the region. Therefore, if the boundary of any cycle of a simple region with holes intersects the boundary of any cycle of a second simple region with holes, then the boundaries of the two regions intersect. There are no special cases for boundaries of hole cycles or outer cycles.

$(v)$ The boundary of $A$ intersects the exterior of $B$ if the boundary of any cycle in $A$ intersects the exterior of the simple region defined by the outer cycle of $B$, or the interior of the simple region defined by any hole cycle of $B$. This follows from the fact that the exterior of a simple region with holes consists of the exterior of the simple region defined by its outer cycle and the interiors of all simple regions defined by its hole cycles, and the fact that the boundary of a simple region with holes consists of the boundaries of all cycles in the region.

At this point, we can determine the 9IM, and hence the topological relationship, between two simple regions with holes by examining the interactions of the simple regions defined by their outer and hole cycles. However, the goal of this paper is to characterize these topological relationships by using topological predicates between simple regions. We now define a method to do this. By examining Theorem 1, it is clear that we need to know all interactions between the simple regions defined by all cycles of two simple regions with holes $A$ and $B$ in order to determine their 9IM. Therefore, we must represent the topological predicates that hold between the simple regions that define the outer cycles of $A$ and $B$, hole cycles of $A$ and $B$, the outer cycle of $A$ and all hole cycles of $B$, and the outer cycle of $B$ and all hole cycles $A$. If this information is known, then (based on the 9IMs between simple regions) we can directly determine the values for parts $(i),(i i),(i v)$, and $(v)$ from Theorem 1. This follows from the fact that each of these parts is defined based on the existence or non-existence of certain predicates holding between pairs of components from two simple regions with holes. However, knowing which predicates hold between the above combinations is insufficient to determine the value for part (iii) of Theorem 1. This is because the second part of the conjunction requires knowledge of the existence of a certain topological configuration between three components of simple regions with holes (namely that there exists a hole in one simple region with holes that is contained in the interior of the outer cycle of a second simple region with holes, and is not contained in a hole of the second simple region with holes). Therefore, in order to determine the topological relationship between two simple regions with holes $A$ and $B$ based on topological predicates between simple regions, we must discover all the topological predicates that hold between the simple regions representing the outer cycles from $A$ and $B$, the outer cycle from $A$ and all hole cycles from $B$, the outer cycle from $B$ and all hole cycles from $A$, and all hole cycles from $A$ and all hole cycles from $B$. Furthermore, we must indicate whether the special situation in part (iii) of Theorem 1 holds. Thus, we characterize a topological relationship between two simple regions with holes as a component based topological relationship (CBTR), which consists of four sets of topological predicates, contain- 
ing all topological predicates that exist between the outer cycle of one region and the outer cycle of the other, the outer cycle of one region and all hole cycles of the other, etc, and two boolean values indicating whether the special situation for part (iii) of Theorem 1 holds between each $A$ and $B$ and between $B$ and $A$. We formally define the CBTR between two simple regions with holes as:

Definition 1. Let $A$ and $B$ be two simple regions with holes and $P_{S R}$ be the set of topological predicates between simple regions. The CBTR that describes their topological relationship is a six-tuple $\mathrm{CBTR}=\left(\mathrm{OO}, \mathrm{OH}, \mathrm{HO}, \mathrm{HH}, \mathrm{B}_{\mathrm{HHO}}, \mathrm{B}_{\mathrm{OHH}}\right)$ defined as:

$$
\begin{aligned}
& O O=\left\{p \in P_{S R} \mid \exists c \in \omega(A), \exists d \in \omega(B): p(c, d)\right\} \\
& O H=\left\{p \in P_{S R} \mid \exists c \in \omega(A), \exists j \in \mathfrak{l}(B): p(c, j)\right\} \\
& H O=\left\{p \in P_{S R} \mid \exists h \in \mathfrak{l}(A), \exists d \in \omega(B): p(h, d)\right\} \\
& H H=\left\{p \in P_{S R} \mid \exists h \in \mathfrak{l}(A), \exists j \in \mathfrak{l}(B): p(h, j)\right\} \\
& B_{O H H}=(\exists j \in \mathfrak{l}(B), \exists h \in \mathfrak{l}(A), \exists c \in \omega(A):(\operatorname{equal}(j, c) \\
&\vee \operatorname{coveredBy}(j, c) \vee \operatorname{inside}(j, c)) \\
&\wedge \neg(\text { equal }(j, h) \vee \operatorname{coveredBy}(j, h), \operatorname{inside}(j, h))) \\
& B_{H H O}=(\exists h \in \mathfrak{l}(A), \exists j \in \mathfrak{l}(B), \exists d \in \omega(B):(\operatorname{equal}(h, d) \\
&\vee \operatorname{coveredBy}(h, d) \vee \operatorname{inside}(h, d)) \\
&\wedge \neg(\text { equal }(h, j) \vee \operatorname{coveredBy}(h, j), \operatorname{inside}(h, j)))
\end{aligned}
$$

We are now able to compute a CBTR between any two simple regions with holes by using only topological relationships between simple regions. Furthermore, because the topological relationships between simple regions are known, it is clear that given a CBTR between two simple regions with holes, we can derive the 9IM between simple regions with holes from it based on Theorem 1 . To do this, we need to convert our characterizations in Theorem 1 such that each of the entries is defined based on topological relationships between simple regions. Using the 9IM of the 8 topological relationships between simple regions (Table 1) and Theorem 1, we obtain the following characterization of the 9IM entries for simple region with holes.

Corollary 1. Let $A$ and $B$ be two simple regions with holes. Let $p$ be a topological relationship between simple regions. We denote $p_{O O}(A, B), p_{O H}(A, B), p_{H O}(A, B)$, and $p_{H H}(A, B)$ as the topological relationship between the outer cycles of $A$ and $B$, the outer cycle of $A$ and a hole of $B$, a hole of $A$ and the outer cycle of $B$, and a hole of $A$ and a hole of $B$ respectively. The values of the entries in the 9IM describing the topological relationship between $A$ and $B$ can now be written as: 
(i) $M_{(A, B)}{ }^{\circ \circ}=\left(\right.$ equal $_{O O}(A, B) \vee$ inside $_{O O}(A, B) \vee$ covered $y_{O o}(A, B)$

$\vee$ contains $_{O O}(A, B) \vee$ coversoo $_{O}(A, B) \vee$ overlap $\left._{O O}(A, B)\right)$

$\wedge \neg$ equal $_{O H}(A, B) \wedge$ inside $_{O H}(A, B) \wedge \neg$ coveredBy $_{O H}(A, B)$

$\wedge \neg$ equal $_{\mathrm{HO}}(A, B) \wedge \neg$ contains $_{\mathrm{HO}}(A, B) \wedge \neg \operatorname{covers}_{\mathrm{HO}}(A, B)$

(ii) $M_{(A, B)}{ }^{\circ} \partial=\left(\left(\right.\right.$ contains $_{O O}(A, B) \vee$ covers $_{O O}(A, B) \vee$ overlapoo $\left._{O}(A, B)\right)$

$\wedge \neg \operatorname{cqual}_{\mathrm{HO}}(A, B) \wedge \neg$ contains $\left._{\mathrm{HO}}(A, B) \wedge \neg \operatorname{covers}_{\mathrm{HO}}(A, B)\right)$

$\vee\left(\left(\right.\right.$ contains $_{O H}(A, B) \vee$ covers $_{O H}(A, B) \vee$ overlap $\left._{O H}(A, B)\right)$

$\wedge \neg \operatorname{equal}_{H H}(A, B) \wedge \neg$ contains $\left._{H H}(A, B) \wedge \neg \operatorname{covers}_{H H}(A, B)\right)$

(iii) $M_{(A, B)}{ }^{\circ-}=$ disjointoo $(A, B) \vee$ meetoo $(A, B) \vee$ containsoo $(A, B)$

$\vee$ coversoo $(A, B) \vee$ overlapoo $(A, B) \vee B_{O H H}$

(iv) $M_{(A, B)} \partial \partial=$ meet oo $(A, B) \vee$ equal $_{O o}(A, B) \vee$ coveredByoo $(A, B)$

$\vee$ covers $_{O O}(A, B) \vee$ overlapoo $(A, B) \vee$ meet $_{O H}(A, B)$

$\vee$ equal $_{O H}(A, B) \vee$ covered $y_{O H}(A, B) \vee$ covers $_{O H}(A, B)$

$\vee$ overlap $_{O H}(A, B) \vee$ meet $_{H O}(A, B) \vee$ equal $_{H O}(A, B)$

$\vee$ coveredBy $_{H O}(A, B) \vee$ covers $_{H O}(A, B) \vee$ overlap $_{H O}(A, B)$

$\vee$ meet $_{H H}(A, B) \vee$ equal $_{H H}(A, B) \vee$ covered $y_{H H}(A, B)$

$\vee$ covers $_{H H}(A, B) \vee$ overlap $_{H H}(A, B)$

(v) $M_{(A, B)} \partial^{-}=$disjoint $_{O O}(A, B) \vee$ meet $_{O O}(A, B) \vee$ contains $_{O O}(A, B)$

$\vee$ covers $_{O O}(A, B) \vee$ overlapoo $_{O}(A, B) \vee$ disjoint $_{H O}(A, B)$

$\vee$ meet $_{\mathrm{HO}}(A, B) \vee$ contains $_{\mathrm{HO}}(A, B) \vee$ covers $_{\mathrm{HO}}(A, B)$

$\vee$ overlap $_{H O}(A, B) \vee$ inside $_{O H}(A, B) \vee$ covered $y_{O H}(A, B)$

$\vee$ overlap $_{O H}(A, B) \vee$ inside $_{H H}(A, B) \vee$ coveredBy $_{H H}(A, B)$

$\vee \operatorname{overlap}_{H H}(A, B)$

Given any valid configuration of two simple regions with holes $A$ and $B$, we can compute their CBTR and determine the 9IM representing the relationship between them using Corollary 1. For example, consider two simple regions with holes $A$ and $B$ as shown in Figure 4. $B$ has a single hole covered by $A$ which has no hole. The CBTR for this scene can be computed by using any available implementation of topological relationships between simple regions. We obtain $\operatorname{CBTR}(A, B)=(\{$ inside $\},\{$ covers $\}, \varnothing, \varnothing$, true, false $)$. By applying this CBTR to Corollary 1, we obtain $M_{(A, B)}{ }^{\circ \circ}=$ true; $M_{(A, B)}{ }^{\circ} \partial=$ true; $M_{(A, B)}{ }^{\circ-}=$ true; $M_{(A, B)} \partial^{\circ}=$ true $; M_{(A, B)} \partial \partial=$ true $; M_{(A, B)} \partial^{-}=$false $; M_{(A, B)}{ }^{-\circ}=$ true $; M_{(A, B)}-\partial=$ true; and $M_{(A, B)}{ }^{--}=$true. The 9IM corresponding to the above value of these entries is exactly the same as the 9IM for coversOverfill shown in Table 2 . Therefore, the topological relationship between simple regions with holes $A$ and $B$ is identified as coversOverfill.

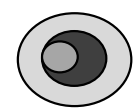

Fig. 4. A simple region with one hole B (light) and a simple region with no hole A (dark) with their shared part shaded the darkest. 


\section{CONCLUSIONS}

Although there has been a large amount of research in modeling topological relationships between regions with holes and complex regions, the implementation of these relationships is generally not as widely available as that of topological relationships between simple regions. In this paper, we have shown how we can derive topological relationships between simple regions with holes using only topological relationships between simple regions. This opens up the possibility of using the simpler and more straight forward implementation of topological relationships between simple regions as the basis for implementing topological relationships between simple regions with holes as well as more complex topological relationships such as local and hybrid topological relationships between complex regions.

\section{References}

Clementini E and Di Felice P (1996) A Model for Representing Topological Relationships between Complex Geometric Features in Spatial Databases. Information Systems, 90:121136.

Clementini E, Di Felice P, and Califano G (1995) Composite Regions in Topological Queries. Information Systems, 20:579-594.

Egenhofer M, Clementini E, and Di Felice P (1994) Topological Relations between Regions with Holes. Int. Journal of Geographical Information Systems, 8:128-142.

Egenhofer MJ, Frank A, and Jackson JP (1989) A Topological Data Model for Spatial Databases. In 1st Int. Symp. on the Design and Implementation of Large Spatial Databases, pages 271-286. Springer-Verlag.

Egenhofer MJ and Franzosa RD (1991) Point-Set Topological Spatial Relations. Int. Journal of Geographical Information Systems, 5:161-174.

Egenhofer MJ and Herring J (1990) Categorizing Binary Topological Relations Between Regions, Lines, and Points in Geographic Databases. Technical report, National Center for Geographic Information and Analysis, University of California, Santa Barbara.

McKenney M, Pauly A, Praing R, and Schneider M (2007) Local Topological Relationships for Complex Regions. In 10th Int. Symp. on Spatial and Temporal Databases, pages 203220.

Schneider M and Behr T (2006) Topological Relationships between Complex Spatial Objects. ACM Trans. on Database Systems, 31:39-81. 